\title{
Cardiovascular disease prediction: do pulmonary disease-related chest CT features have added value?
}

\author{
Pushpa M. Jairam • Pim A. de Jong • Willem P. Th. M. Mali • Ivana Isgum • \\ Yolanda van der Graaf • on behalf of the PROVIDI study-group
}

Received: 23 April 2014 / Revised: 16 September 2014 / Accepted: 10 November 2014 / Published online: 14 March 2015

(C) The Author(s) 2015. This article is published with open access at Springerlink.com

\begin{abstract}
Objective Certain pulmonary diseases are associated with cardiovascular disease (CVD). Therefore we investigated the incremental predictive value of pulmonary, mediastinal and pleural features over cardiovascular imaging findings.

Methods A total of 10,410 patients underwent diagnostic chest $\mathrm{CT}$ for non-cardiovascular indications. Using a casecohort approach, we visually graded CTs from the cases and from an approximately $10 \%$ random sample of the baseline cohort $(n=1,203)$ for cardiovascular, pulmonary, mediastinal and pleural findings. The incremental value of pulmonary disease-related CT findings above cardiovascular imaging findings in cardiovascular event risk prediction was quantified by comparing discrimination and reclassification.

Results During a mean follow-up of 3.7 years (max. 7.0 years), 1,148 CVD events (cases) were identified. Addition of pulmonary, mediastinal and pleural features to a cardiovascular imaging findings-based prediction model led to marginal improvement of discrimination (increase in c-index from $0.72(95 \%$ CI $0.71-0.74)$ to $0.74(95 \%$ CI $0.72-0.75)$ ) and reclassification measures (net reclassification index $6.5 \%$ $(p<0.01))$.
\end{abstract}

Electronic supplementary material The online version of this article (doi:10.1007/s00330-014-3495-0) contains supplementary material, which is available to authorized users.

P. M. Jairam $(\bowtie) \cdot$ Y. van der Graaf

Julius Center for Health Sciences and Primary Care, University

Medical Center Utrecht, Utrecht, The Netherlands

e-mail: pjairam@umcutrecht.nl

P. M. Jairam • P. A. de Jong • W. P. T. M. Mali

Department of Radiology, University Medical Center Utrecht,

Utrecht, The Netherlands

I. Isgum

Image Sciences Institute, University Medical Center Utrecht,

Utrecht, The Netherlands
Conclusion Pulmonary, mediastinal and pleural features have limited predictive value in the identification of subjects at high risk of CVD events beyond cardiovascular findings on diagnostic chest CT scans.

Key Points

- Incidental cardiovascular findings on routine chest CT can predict cardiovascular disease

- Non-cardiovascular chest CT abnormalities are associated with cardiovascular disease

- Non-cardiovascular chest CT features have limited predictive value beyond cardiovascular features

Keywords Chest CT imaging $\cdot$ CVD risk prediction · Pulmonary disease-related CT findings $\cdot$ Cardiovascular imaging $\cdot$ Cardiovascular disease

\section{Introduction}

Chest computed tomography (CT) has emerged as a commonly used imaging modality in the evaluation of thoracic diseases, with more than 10 million chest CTs performed annually in the USA $[1,2]$. In addition to the clinically valuable information embedded in the imaging findings related to the chest $\mathrm{CT}$ indication, incidental findings that are unsuspected or unrelated to the indication contain prognostically valuable information about multiple diseases [3-5]. In fact, in the case of incidental cardiovascular $\mathrm{CT}$ features, such as coronary and extracoronary calcifications, cardiovascular disease (CVD) risk can immediately be derived from these markers of organ damage visible on CT [6-11].

An explorative study [12] has proposed a different approach for CVD risk prediction strictly based on information readily available to the radiologist. The provided prediction rule allows one to calculate up to 5-year predicted probabilities for non-fatal and fatal CVD events by combining age, 
gender, CT indication, cardiac diameter, left anterior descending coronary artery calcifications, mitral valve calcifications and descending aorta calcifications. Addressing conventional risk factors is not possible in daily routine practice, since this information is in general not available to the radiologist. Moreover, risk factor detection, earlier in the disease process, precedes in many patients the presence of the image findings. Additionally, a substantial proportion of CVD events occur in individuals without conventional risk factors or in subjects with yet undetected or underdiagnosed risk factors [13]. There are several studies supporting a shift in CVD risk assessment from conventional risk factors, as they are merely a surrogate for atherosclerosis, to direct measures of subclinical atherosclerosis [13-15]. Utilization of markers of subclinical target organ damage for CVD risk prediction, like cardiovascular CT features incorporated in the imaging-based model, provide a novel strategy and adequate estimation of CVD risk irrespective of the conventional risk factor status.

Emerging data indicate a direct link between certain pulmonary diseases and cardiovascular disease. Mechanisms such as ongoing pulmonary inflammation, repair mechanisms, misbalances between proteases and anti-proteases and autoimmune phenomena have been suggested to explain the relationship between pulmonary diseases and cardiovascular disease. Additionally, chest CT abnormalities reflecting these pulmonary diseases, e.g. airway thickening, pulmonary ground glass opacity, pulmonary consolidation, pleural effusion and mediastinal lymphadenopathy, were found to be associated with CVD [16-19]. Whether these pulmonary, mediastinal and pleural characteristics, which can be evaluated in the same image as the cardiovascular findings without additional examining or radiation exposure, improve CVD risk estimation beyond what is possible with cardiovascular findings is unknown. To address this issue we evaluated the incremental predictive value of pulmonary, mediastinal and pleural findings on routine diagnostic chest CT over a cardiovascular CT features-based prediction model.

\section{Materials and methods}

\section{Study population}

This is an ancillary study of the PROgnostic Value of unrequested Information in Diagnostic Imaging (PROVIDI) study. The PROVIDI study was conceived from the perspective of the radiologists, to determine the prognostic relevance of the growing amount of uncertain incidental findings. Only information readily available to the radiologist is addressed in this study. Since in general, other risk factors besides age and gender are not easy accessible for the radiologist, we could not provide information on the distribution of these other risk factors in the current study. This study was approved by the ethical review board of the University Medical Center Utrecht (number 06/193); informed consent was waived because a privacy protocol was implemented in the study protocol.

The rationale and design of the PROVIDI study has been described in detail [4]. Briefly, the study population comprised 23,443 subjects aged 40 years and over who underwent routine clinical chest CT imaging between 2002 and 2005 for various diagnostic clinical indications in participating hospitals in the Netherlands. Patients with a diagnosis of primary lung cancer (including mesothelioma) or distant metastatic disease from other types of cancer (excluding hematologic malignancies) at baseline were excluded $(n=9,077)$, because of the poor prognosis. Also excluded were patients yielding prior history of CVD or subjects with a CT referral indication directly related to (suspected) cardiovascular pathology $(n=$ $2,303)$, to ensure that the evaluated calcifications were truly "incidental".

\section{Sample selection and study design}

In the present study, 10,410 participants represent the full study cohort and were considered for analyses. Subjects who developed a CVD event during follow-up were identified as cases. We used a case-cohort approach as introduced by Prentice [20], using all cases and an approximately $10 \%$ random sample from the full study cohort (subcohort) at the beginning of the study. With sampling fractions of at least 0.10 , results of a case-cohort analysis are similar to the full study cohort analysis [21]. The cases together with the subcohort define the study population in which the imaging characteristics are determined. The advantage of this design is that it enables survival analyses without the need to score the chest CT images from the full study cohort. Figure 1 shows a flowchart of the study design and the selection of cases and subcohort.

\section{Scoring of CT characteristics}

All chest CT examinations were obtained between January 2002 and December 2005, using multi-detector CT (2-64 detector rows) from different vendors according to the prevailing routine clinical protocols of the participating hospitals. All types of CT (including non-contrast and non-triggered) protocols were considered eligible as long as the field of view included the heart and full length of the thoracic aorta. Section thicknesses varied according to the chest CT indication and corresponding protocol.

CT examinations were examined for left anterior descending (LAD) coronary artery calcifications, mitral valve calcifications, descending aorta calcifications and cardiac diameter. This set of four cardiovascular CT features enabled adequate identification of subjects at high risk of CVD [12]. Additionally, CT images were evaluated for airway thickening, ground 


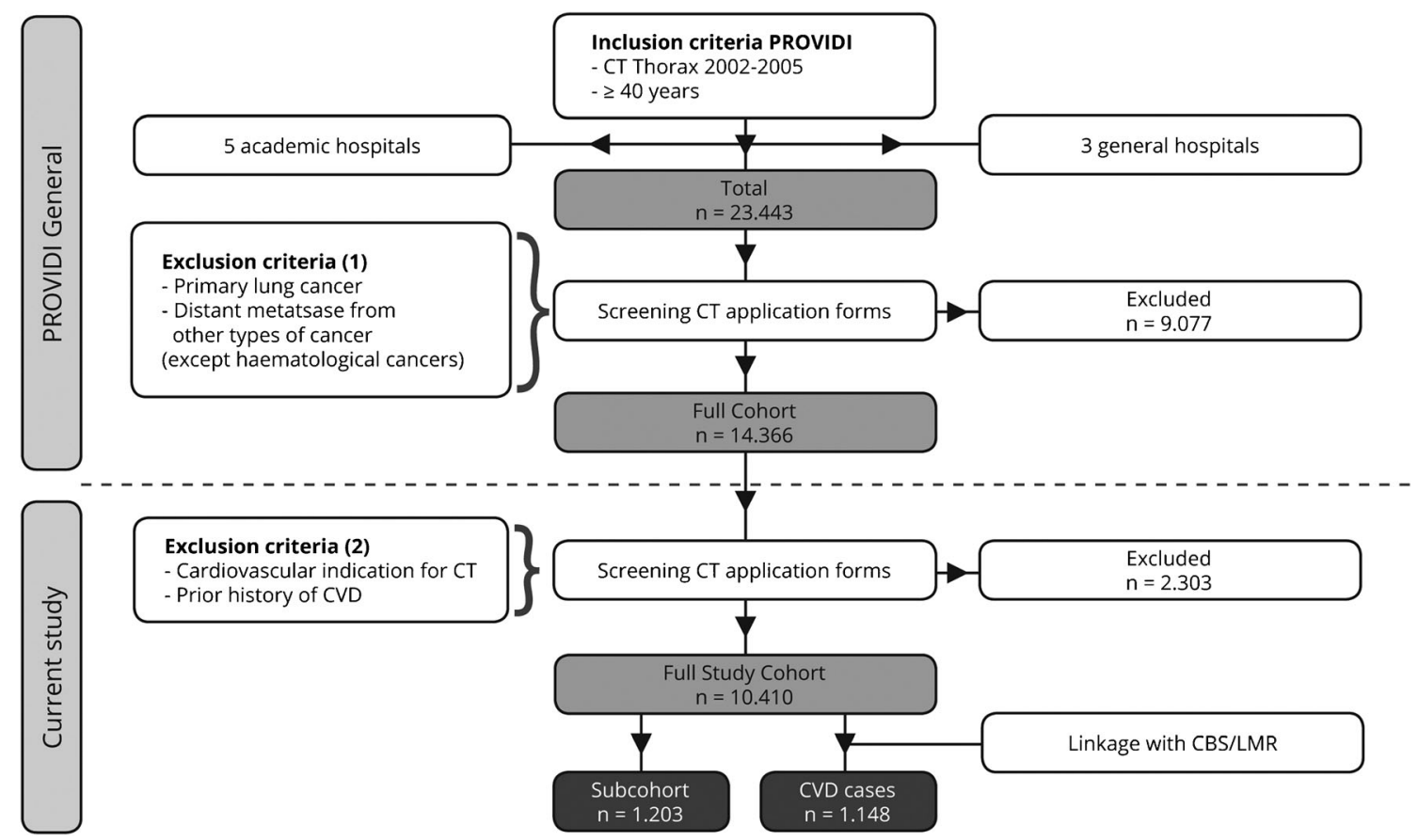

Fig. 1 Flowchart of study design. CBS Central Bureau of Statistics, LMR Dutch medical data registration

glass, consolidation, pleural effusion and mediastinal lymph node diameter. These five pulmonary disease-related CT findings have been previously shown to be associated with future CVD events [19]. The presence and extent of the cardiovascular and pulmonary CT abnormalities were graded using a validated simple ordinal [22] (Table 1). Extensive information about the definitions and grading of the CT features can be found in the Electronic Supplementary Material and examples in Fig. 2.

Follow-up and cardiovascular events

CVD events were defined as all coronary events (myocardial infarction, coronary death, coronary insufficiency and angina), cerebrovascular events (ischaemic stroke, haemorrhagic stroke and transient ischaemic attack), peripheral artery disease (intermittent claudication) and heart failure. Data on fatal and non-fatal CVD events were obtained from the Dutch National Registry of Hospital Discharge Diagnoses and the National Death Registry. This registry assigns a code to each non-fatal and fatal CVD event, according to the International Classification of Diseases, 9th and10th Revision (ICD-9 and ICD-10); details are shown in the Electronic Supplementary Material. The database was linked to the study cohort with a validated probabilistic method [23, 24]. As previously demonstrated, the overall quality of Dutch national registers is high and ICD codes have high sensitivities and positive predictive values $[25,26]$, enabling reliable identification of cases and follow-up. Whenever multiple events occurred, the first event was taken as an end point.

\section{Statistical analyses}

Baseline characteristics and chest CT findings were summarized for the subcohort and the cases. Quantitative data were described with median (interquartile range) because of their abnormal distribution. Qualitative data were described using percentages. To compare subjects within the subcohort and patients who experienced a CVD event, differences in continuous variables were analysed with Mann-Whitney $U$ test. Differences in categorical variables were tested using chi-square tests. The analysis comprised three stages. The first was an assessment of the associations between each pulmonary disease-related $\mathrm{CT}$ finding and future CVD events; the second involved the derivation of a cardiopulmonary $\mathrm{CT}$ features prediction rule consisting of the four graded cardiovascular CT features, plus a selection of pulmonary disease-related CT findings (a forward stepwise procedure was used for predictor selection) and the third involved comparison of discrimination and reclassification between the cardiopulmonary CT features model and the model only based on cardiovascular $\mathrm{CT}$ features. For a more detailed statistical description, see the Electronic Supplementary Material. Analyses were performed with R-project software package, version 2.15 (www.r-project.org). 
Table 1 Semi-quantitative grading score of various cardiovascular and pulmonary disease-related CT findings on chest CT

\begin{tabular}{|c|c|c|c|c|}
\hline & $0=\mathrm{Absent}$ & 1=Mild & $2=$ Moderate & $3=$ Severe \\
\hline \multicolumn{5}{|l|}{ LAD coronary artery calcifications } \\
\hline Number and size of calcifications & None & $\begin{array}{l}1-2 \text { focal limited } \\
\text { to } \leq 2 \text { slices }\end{array}$ & $\begin{array}{l}>2 \text { focal or } 1 \text { extending } \\
>2 \text { slices }\end{array}$ & $\begin{array}{l}\text { Fully calcified coronary } \\
\text { artery extending } \\
\text { multiple slices }\end{array}$ \\
\hline \multicolumn{5}{|l|}{ Descending aorta calcifications } \\
\hline Number and size of calcifications & None & $\leq 3$ focal & $\begin{array}{l}4-5 \text { focal or } 1 \text { extending } \\
\geq 3 \text { slices }\end{array}$ & $\begin{array}{l}>5 \text { focal or }>1 \text { extending } \\
\quad \geq 3 \text { slices }\end{array}$ \\
\hline \multicolumn{5}{|l|}{ Mitral valve calcifications } \\
\hline Number of affected valve leaflet(s) & None & 1 leaflet & 2 leaflets & \\
\hline \multicolumn{5}{|l|}{ Airway thickening } \\
\hline Number of affected lobes (range $0-5$ ) & 0 & 1 & 2 & $\geq 3$ \\
\hline \multicolumn{5}{|l|}{ Consolidation } \\
\hline Number of affected lobes (range $0-5$ ) & 0 & 1 & 2 & $\geq 3$ \\
\hline \multicolumn{5}{|l|}{ Ground glass } \\
\hline Number of affected lobes (range $0-5$ ) & 0 & 1 & 2 & $\geq 3$ \\
\hline \multicolumn{5}{|l|}{ Pleural effusion } \\
\hline $\begin{array}{l}\text { Sum of left and right fluid layers thickness on } \\
\text { axial images, cm }\end{array}$ & 0 & $<6$ & $6-12$ & $>12$ \\
\hline \multicolumn{5}{|l|}{ Lymph node } \\
\hline Short axis diameter of the largest lymph node, $\mathrm{mm}$ & $<6$ & $6-10$ & $>10$ & \\
\hline
\end{tabular}

$L A D$ left anterior descending

Fig. 2 Examples of pulmonary, mediastinal and pleural CT imaging characteristics. a Airway thickening (white arrow) and ground glass in the left lower lobe (black arrows). b Consolidation in the right under lobe (black arrow). c Short axis diameter measurement of the largest mediastinal lymph node. d Pleural effusion measurement of left-sided fluid layer on axial image
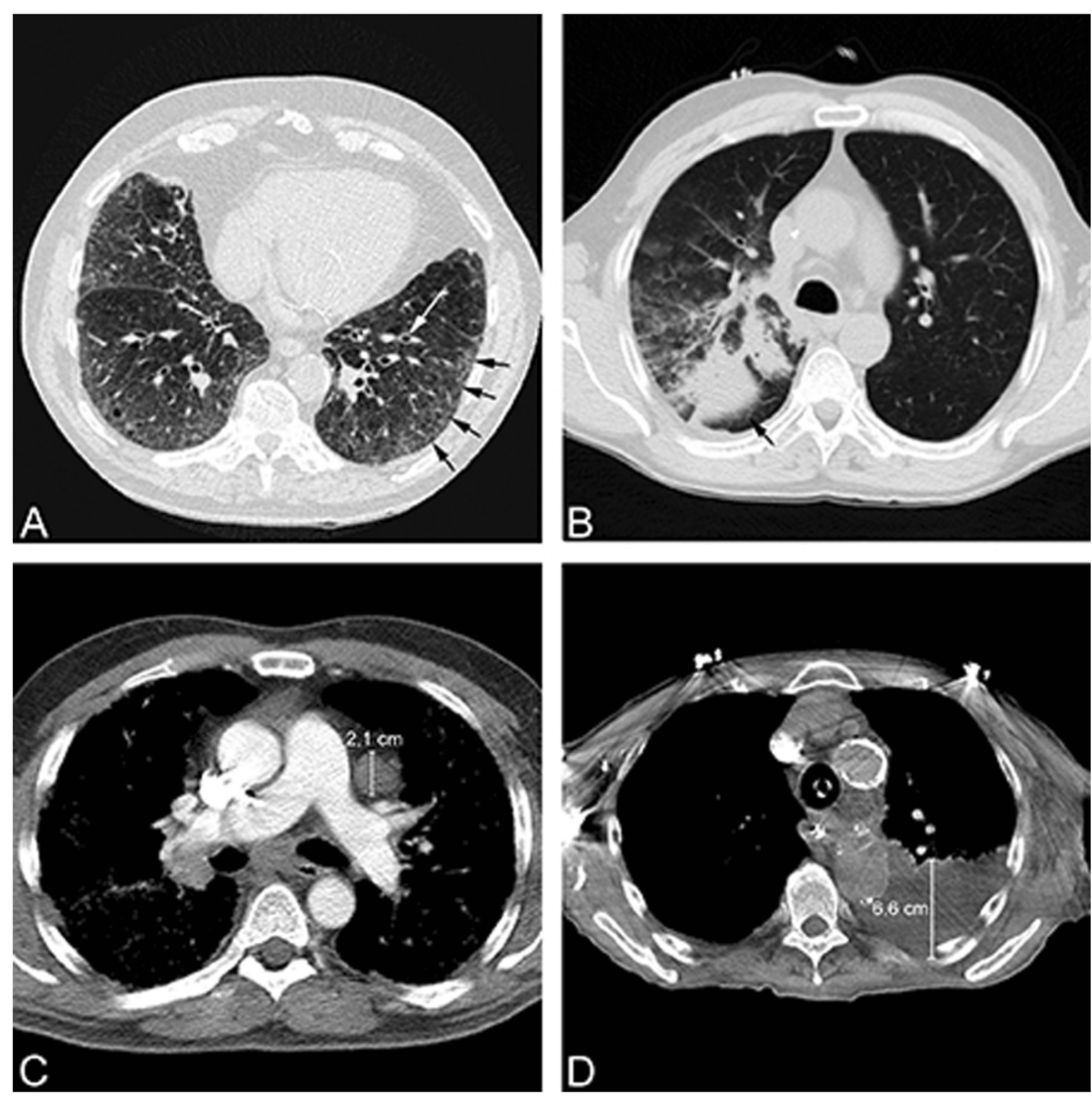


\section{Results}

During a median follow-up of 3.7 years (range 3.2-7.0 years), 1,148 CVD events occurred among the 10,410 subjects of the baseline cohort. Table 2 presents the baseline characteristics and chest CT imaging findings for the subcohort and CVD cases. As expected, the cases were slightly older, more often male and had more numerous and more severe cardiovascular calcifications as well as pulmonary, mediastinal and pleural abnormalities compared to the subcohort. These differences were all statistically significant $(p<0.05)$.

In Table 3, unadjusted and adjusted hazard ratios (HRs) and their $95 \%$ confidence intervals (CIs) for CVD events are presented for the mild, moderate and severe categories of airway thickening, ground glass, consolidation, pleural effusion as well as lymph node diameter compared with the absent category. All five pulmonary, mediastinal and pleural findings were significantly related to CVD risk in the age- and sexadjusted analysis. When estimates were fully adjusted for age, gender, CT indication, LAD coronary artery calcifications, mitral valve calcifications and descending aorta calcifications and cardiac diameter, the association between airway thickening and consolidations with CVD events was no longer significant. Pulmonary, mediastinal and pleural findings that were independently related to future CVD events after accounting for the predictors included in the cardiovascular CT features-based model were ground glass, pleural effusion and lymph node diameter. The HRs for comparison of the severe versus the absent category for these CT features were as follows: ground glass, HR 2.0 (95 \% CI 1.6-2.4); pleural effusion, HR 1.7 (95\% CI 1.3-2.2) and lymph node diameter, HR 1.5 (95\% CI 1.3-1.8) (Fig. 3).

To assess the incremental value of pulmonary diseaserelated CT features over cardiovascular imaging findings, first a Cox proportional hazard model including limited patient characteristics and cardiovascular CT findings was prespecified. This model will further be referred to as the "cardiovascular CT features model" and comprised the following predictors: age, gender, CT indication, cardiac diameter, LAD coronary artery calcifications, mitral valve calcifications and descending aorta calcifications. Second, a "cardiopulmonary CT features model" was derived, adding the three strongest and independent predictors by Cox proportional hazard models of single pulmonary disease-related CT features with future CVD events (ground glass, pleural effusion and lymphadenopathy) to the cardiovascular CT features model. The same selection of the best predictors was obtained with the use of a forward stepwise procedure, for details see the Electronic Supplementary Material.

Addition of ground glass, pleural effusion and lymph node diameter to the cardiovascular CT features model led to marginal improvement of discrimination and reclassification measures (Table 4). Discrimination, expressed by the c-index,
Table 2 Baseline characteristics and chest CT imaging findings for subjects in the subcohort $(n=1,203)$ and for the cardiovascular disease cases $(n=1,148)$

\begin{tabular}{|c|c|c|}
\hline & $\begin{array}{l}\text { Subcohort } \\
n=1,203\end{array}$ & $\begin{array}{l}\text { CVD cases } \\
n=1,148\end{array}$ \\
\hline Age, years & $61(52-71)$ & $68(59-75)$ \\
\hline Male gender, $n(\%)$ & $698(58)$ & $723(63)$ \\
\hline \multicolumn{3}{|l|}{ CT indication, $n(\%)$} \\
\hline Pulmonary disease & $457(38)$ & $505(44)$ \\
\hline Haematological malignancy & $132(11)$ & $80(7)$ \\
\hline Mediastinal disease & $132(11)$ & $103(9)$ \\
\hline Rules out lung cancer & $277(23)$ & $241(21)$ \\
\hline Pulmonary embolism & $72(6)$ & $80(7)$ \\
\hline Other & $144(12)$ & $126(11)$ \\
\hline \multicolumn{3}{|c|}{ LAD coronary artery calcifications, $n(\%)$} \\
\hline Mild & $373(31)$ & $298(26)$ \\
\hline Moderate & $229(19)$ & $321(28)$ \\
\hline Severe & $120(10)$ & $207(18)$ \\
\hline \multicolumn{3}{|c|}{ Descending aorta calcifications, $n(\%)$} \\
\hline Mild & $325(27)$ & $333(29)$ \\
\hline Moderate & $180(15)$ & $253(22)$ \\
\hline Severe & $72(6)$ & $195(17)$ \\
\hline \multicolumn{3}{|l|}{ Mitral valve calcification, $n(\%)$} \\
\hline 1 leaflet & $84(7)$ & $149(13)$ \\
\hline 2 leaflets & $12(1)$ & $34(3)$ \\
\hline Cardiac diameter, mm & $125(115-134)$ & $130(121-141)$ \\
\hline \multicolumn{3}{|l|}{ Airway thickening, $n(\%)$} \\
\hline Mild & $120(10)$ & $138(12)$ \\
\hline Moderate & $132(11)$ & $161(14)$ \\
\hline Severe & $108(9)$ & $138(12)$ \\
\hline \multicolumn{3}{|l|}{ Ground glass, $n(\%)$} \\
\hline Mild & $144(12)$ & $172(15)$ \\
\hline Moderate & $84(7)$ & $149(13)$ \\
\hline Severe & $96(8)$ & $138(12)$ \\
\hline \multicolumn{3}{|l|}{ Consolidation, $n(\%)$} \\
\hline Mild & $180(15)$ & $184(16)$ \\
\hline Moderate & $108(9)$ & $126(11)$ \\
\hline Severe & $48(4)$ & $57(5)$ \\
\hline \multicolumn{3}{|l|}{ Pleural effusion, $n(\%)$} \\
\hline Mild & $60(5)$ & $57(5)$ \\
\hline Moderate & $72(6)$ & $115(10)$ \\
\hline Severe & $48(4)$ & $92(8)$ \\
\hline \multicolumn{3}{|l|}{ Lymph node diameter, $n(\%)$} \\
\hline 6-10 mm & $457(38)$ & $551(48)$ \\
\hline$>10 \mathrm{~mm}$ & $252(21)$ & $287(25)$ \\
\hline
\end{tabular}

Values are mean \pm standard deviation, median (interquartile range) or $n$ (\%)

$L A D$ left anterior descending

increased from $0.72(95 \%$ CI $0.71-0.74)$ to $0.74(95 \% \mathrm{CI}$ $0.72-0.75)$. Reclassification as measured by the net 
Table 3 Hazard ratios (95\% CI) for cardiovascular events $(n=1,148)$ during a median follow-up period time of 3.7 years according to the pulmonary disease-related CT findings on routine chest CT

\begin{tabular}{|c|c|c|}
\hline & $\begin{array}{l}\text { Sex- and age-adjusted } \\
\text { HR }(95 \% \mathrm{CI})\end{array}$ & $\begin{array}{l}\text { Multivariable }{ }^{\mathrm{a}} \text { adjusted } \\
\text { HR }(95 \% \mathrm{CI})\end{array}$ \\
\hline \multicolumn{3}{|c|}{ Airway thickening (\%) } \\
\hline Absent & Reference & Reference \\
\hline Mild & $1.3(1.3-1.5)$ & $1.0(0.8-1.3)$ \\
\hline Moderate & $1.3(1.1-1.5)$ & $1.2(1.0-1.4)$ \\
\hline Severe & $1.4(1.1-1.7)$ & $1.1(0.9-1.3)$ \\
\hline \multicolumn{3}{|c|}{ Consolidation (\%) } \\
\hline Absent & Reference & Reference \\
\hline Mild & $1.1(0.9-1.3)$ & $0.9(0.7-1.1)$ \\
\hline Moderate & $1.5(1.2-1.8)$ & $1.2(1.0-1.5)$ \\
\hline Severe & $1.5(1.1-1.9)$ & $1.3(1.0-1.7)$ \\
\hline \multicolumn{3}{|c|}{ Ground glass (\%) } \\
\hline Absent & Reference & Reference \\
\hline Mild & $1.4(1.2-1.7)$ & $1.6(1.3-1.8)$ \\
\hline Moderate & $1.9(1.4-2.6)$ & $2.0(1.7-2.4)$ \\
\hline Severe & $2.2(1.6-3.0)$ & $2.0(1.6-2.4)$ \\
\hline \multicolumn{3}{|c|}{ Pleural effusion (\%) } \\
\hline Absent & Reference & Reference \\
\hline Mild & $1.3(1.0-1.7)$ & $1.0(0.8-1.3)$ \\
\hline Moderate & $2.7(1.8-4.0)$ & $1.7(1.4-2.2)$ \\
\hline Severe & $2.3(1.5-3.5)$ & $1.7(1.3-2.2)$ \\
\hline \multicolumn{3}{|c|}{ Lymph node diameter (mm) } \\
\hline$<6$ & Reference & Reference \\
\hline $6-10$ & $1.8(1.5-2.0)$ & $1.5(1.3-1.8)$ \\
\hline$>10$ & $1.9(1.6-2.2)$ & $1.5(1.3-1.8)$ \\
\hline
\end{tabular}

${ }^{\text {a }}$ Multivariable adjusted $=$ adjustment for predictors of the cardiovascular CT features model: age, gender, CT indication, left anterior descending coronary artery calcification, mitral valve calcification, descending aortic calcification and the cardiac diameter

reclassification improvement (NRI) was $6.5 \%(P<0.01)$. This indicates that with the cardiopulmonary $\mathrm{CT}$ features model $6.5 \%$ of the subjects are more appropriately reclassified compared with the cardiovascular CT features model, with $0 \%$ improvement in cases and $6.5 \%$ in noncases.

\section{Discussion}

In this case-cohort study of 10,410 subjects who underwent routine clinical chest CT scanning, the incremental value of pulmonary, mediastinal and pleural findings over certain cardiovascular characteristics in CVD event risk prediction was evaluated. Ground glass, pleural effusion and lymphadenopathy were associated with an increased risk of CVD events, independent of age, gender, CT indication, cardiac diameter, LAD coronary artery calcifications, mitral valve calcifications and descending aorta calcifications. However, improvement of CVD event risk prediction beyond cardiovascular imaging findings with addition of these three pulmonary, mediastinal and pleural imaging features was only marginal. Thus, incidental vascular calcifications alone offer an important opportunity to identify patients at high risk of cardiovascular disease. Therefore we recommend to routinely report these findings to allow further work-up and/or preventative treatment. This can be an efficient strategy to effectuate a further decline of the global cardiovascular disease burden without providing additional radiation to the patient or increasing health-care costs. However, in many institutions, there still is a controversy among radiologists whether incidental findings should be reported. Correspondingly, a professional consensus about standardized and universal reporting of incidental vascular calcification in routine radiological practice should be discussed and further developed.

Our data provide evidence for the established direct association between certain pulmonary diseases and CVD [16-19], as airway thickening, ground glass, consolidation, pleural effusion and lymphadenopathy were demonstrated to be associated with CVD risk. This positive relation can be explained because an important mutual cause for these imaging findings, inflammation, has a central role in the atherosclerotic process precipitating CVD events [27-30]. Consistent with our findings, markers of inflammation measurable in serum or plasma (e.g. high sensitivity C-reactive protein, interleukin 6, fibrinogen) have also been associated with CVD risk [31]. Additionally, some abnormalities reflect (subclinical) heart failure. The most common cause of bilateral pleural effusion is heart failure [27]. Bilateral effusion can be a direct marker of CVD, explaining the significant association between pleural effusion and CVD risk even after adjustment for cardiovascular imaging features.

When adjusted for cardiovascular imaging features, only ground glass, pleural effusion and lymphadenopathy were demonstrated to be independently associated with CVD event risk. This may indicate that these pulmonary disease-related CT findings exert their hazardous effect on future CVD events through other pathways than those through which cardiovascular findings affect CVD risk. However, as these abnormalities may reflect (subclinical) heart failure it could well be that they are merely a consequence of the disease. Ground glass, pleural effusion and lymphadenopathy were selected for addition to the cardiovascular CT features model as they were independently associated with CVD. Using a forward stepwise procedure resulted in identical predictor selection. Although these features were independently associated with the risk of later CVD events, their incremental value beyond cardiovascular findings was marginal. Addition of these three imaging features to the cardiovascular CT features model 


\section{CT - imaging Finding}

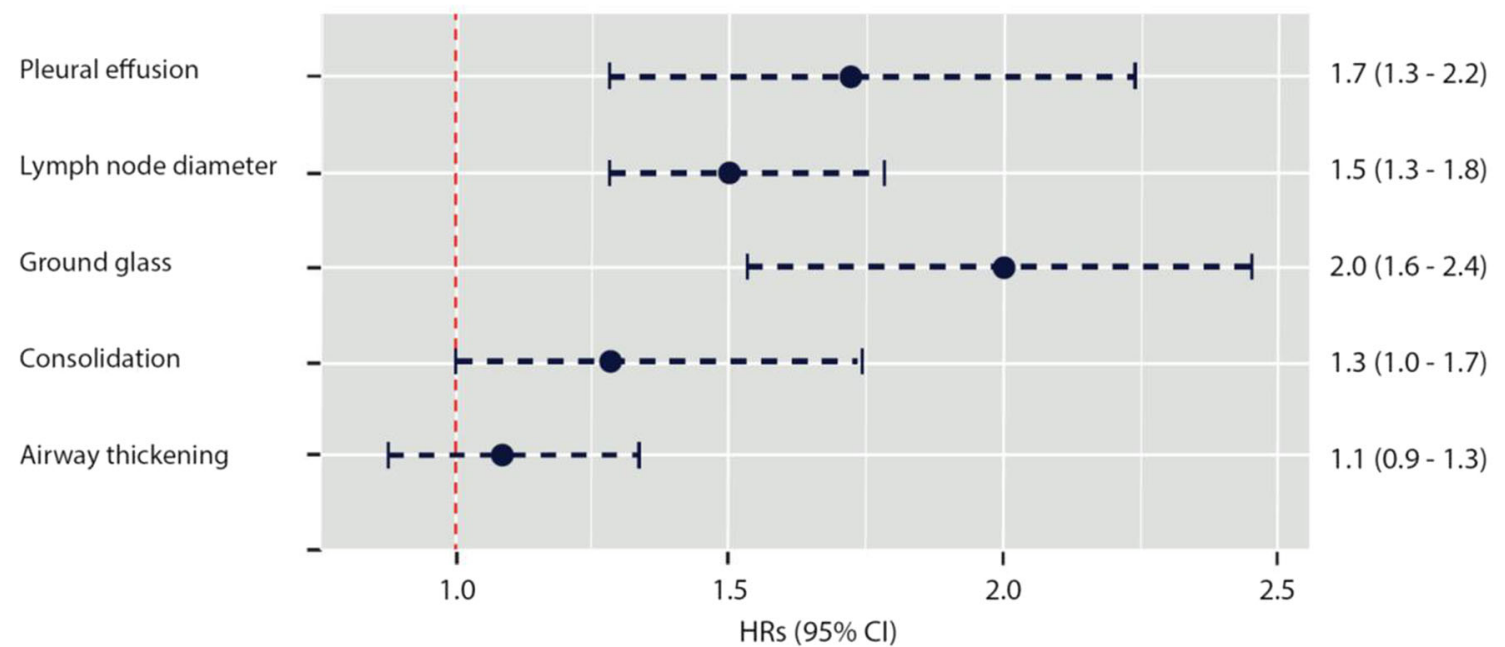

Fig. 3 Multivariable-adjusted hazard ratios (HRs) and $95 \%$ confidence interval $(95 \% \mathrm{CI})$ for cardiovascular events comparing the severe versus the absent category of pleural effusion (HR 1.7 [95\% CI 1.3-2.2]), lymph node diameter (HR 1.5 [95 \% CI 1.3-1.8]), airway thickening (HR 1.1 [95 \% CI 0.9-1.3]), consolidation (HR 1.3 [95\% CI 1.0-1.7] and ground glass (HR 2.0 [95 \% CI 1.6-2.4]) yielded an NRI in the intermediate-risk group of less than $10 \%$, falling below reclassification thresholds that are considered clinically relevant [32].

\section{Limitations}

One of the limitations of our study that merits consideration is that our cohort was comprised of a population of Caucasian Dutch descent. Therefore the generalizability of our findings to populations of different racial or ethnic backgrounds remains uncertain. Another issue that merits consideration is that assessment of pulmonary CT findings like consolidations, ground glass and airway thickening had a relatively low interreader reliability. This may have influenced the association between these findings with CVD events. Moreover, we favoured not to use expert cardiothoracic radiologists as in our practice most chest CT images are interpreted by general radiologists, and often the lungs are read by cardiac radiologists and the arteries by chest radiologists. We think that the quality of the readers will have minimally affected the results. Additionally, the CT examinations varied in slice thickness, use of contrast medium and CT machine vendors. This may have given rise to the possibility of missing subtle CT findings like small coronary artery calcification, bronchial wall thickening and ground glass opacity. However, our visual assessment of the CT findings is a real practice scenario, where visual assessment based on a variety of protocols, also compared to quantitative assessment, has been demonstrated to be relatively simple, inexpensive and independent of machine and reconstruction algorithms [33]. Lastly, in prognostic research, candidate predictors should be measured using methods easily applicable-or potentially applicable-to daily practice [34].

Table 4 Discrimination and reclassification estimates for cardiovascular events ( $n=1,148$ events) during a median follow-up period time of 3.7 years according to the cardiovascular CT features model without and with addition of pulmonary, mediastinal and pleural chest CT findings

\begin{tabular}{lll}
\hline & Cardiovascular CT features model $^{\mathrm{c}}$ & Cardiopulmonary CT features model $^{\mathrm{d}}$ \\
\hline C-statistic $(95 \% \mathrm{CI})^{\mathrm{a}}$ & $0.72(0.71-0.74)$ & $0.74(0.72-0.75)$ \\
Net Reclassification Index (NRI) $(95 \% \mathrm{CI})^{\mathrm{b}}$ & NA & $6.50(4.44-8.55)$ \\
\hline
\end{tabular}

${ }^{\text {a }} \mathrm{C}$-statistic is corrected for over optimism by using 150 bootstrap repetitions

b Percentage of NRI $(95 \% \mathrm{CI})$ for the cardiovascular CT features model versus the cardiopulmonary CT features model, using 5-year predicted cardiovascular risks and risk categories of $<5 \%, 5-10 \%$ and $>10 \%$

${ }^{\mathrm{c}}$ Cardiovascular CT features model included the following variables: age, gender, CT indication, left anterior descending coronary artery calcification, mitral valve calcification, descending aortic calcification and the cardiac diameter

d Cardiopulmonary CT features model included the following variables: age, gender, CT indication, left anterior descending coronary artery calcification, mitral valve calcification, descending aortic calcification and the cardiac diameter plus ground glass, pleural effusion and lymph node diameter 


\section{Conclusion}

Ground glass, pleural effusion and lymphadenopathy on routine clinical CT examinations were independent predictors of future CVD events. However, addition of these imaging features to a risk model based on cardiovascular findings only marginally improves 5-year CVD event risk prediction in a Caucasian Dutch population. Pulmonary, mediastinal and pleural features have limited predictive value in the identification of subjects at high risk of CVD events beyond cardiovascular findings on diagnostic chest CTs.

Acknowledgments The current study is a part of the PROVIDI study and is funded by the Netherlands Organization for Scientific ResearchMedical Sciences (NWO-MW), grant 40-00812-98-07-005, no relationship with industry exists.

The scientific guarantor of this publication is Prof. Dr. Y. van der Graaf. The authors of this manuscript declare no relationships with any companies whose products or services may be related to the subject matter of the article. This study has received funding by the Netherlands Organization for Scientific Research-Medical Sciences (NWO-MW), grant 40-00812-98-07-005, no relationship with industry exists. Prof. Dr. van der Graaf has significant statistical expertise as she is Professor of Clinical Epidemiology at the Julius Center for Health Sciences and Primary Care of University Medical Center Utrecht. Dr. Jairam has significant statistical expertise as she is a clinical epidemiologist. Institutional review board approval was approved by the ethical review board of the University Medical Center Utrecht (number 06/193), informed consent was waived because a privacy protocol was implemented in the study protocol. Written informed consent was waived by the institutional review board.

Open Access This article is distributed under the terms of the Creative Commons Attribution Noncommercial License which permits any noncommercial use, distribution, and reproduction in any medium, provided the original author(s) and the source are credited.

\section{References}

1. Johannessen A, Skorge TD, Bottai M, Grydeland TB, Nilsen RM et al (2013) Mortality by level of emphysema and airway wall thickness. Am J Respir Crit Care Med 187:602-608

2. Brenner DJ (2012) Radiation and chest CT scans: are there problems? What should we do? Chest 142:549-550

3. Mets OM, de Jong PA, Prokop M (2012) Computed tomographic screening for lung cancer: an opportunity to evaluate other diseases. JAMA 308:1433-1434

4. Gondrie MJ, Mali WP, Buckens CF, Jacobs PC, Grobbee DE et al (2010) The PROgnostic Value of unrequested Information in Diagnostic Imaging (PROVIDI) study: rationale and design. Eur J Epidemiol 25:751-758

5. Alpert JB, Naidich DP (2011) Imaging of incidental findings on thoracic computed tomography. Radiol Clin N Am 49:267-289

6. Gondrie MJ, Mali WP, Jacobs PC, Oen AL, van der Graaf Y (2010) Cardiovascular disease: prediction with ancillary aortic findings on chest CT scans in routine practice. Radiology 257:549-559

7. Gondrie MJ, van der Graaf Y, Jacobs PC, Buckens SC, Mali WP (2011) The prognostic value of vascular diameter measurements on routine chest computed tomography in patients not referred for cardiovascular indications. J Comput Assist Tomogr 35:734-741

8. Gondrie MJ, van der Graaf Y, Jacobs PC, Oen AL, Mali WP (2011) The association of incidentally detected heart valve calcification with future cardiovascular events. Eur Radiol 21:963-973

9. Jacobs PC, Gondrie MJ, Mali WP, Oen AL, Prokop M et al (2011) Unrequested information from routine diagnostic chest $\mathrm{CT}$ predicts future cardiovascular events. Eur Radiol 21:1577-1585

10. Nasir K, Katz R, Al-Mallah M, Takasu J, Shavelle DM et al (2010) Relationship of aortic valve calcification with coronary artery calcium severity: the Multi-Ethnic Study of Atherosclerosis (MESA). J Cardiovasc Comput Tomogr 4:41-46

11. Nasir K, Katz R, Takasu J, Shavelle DM, Detrano R et al (2008) Ethnic differences between extra-coronary measures on cardiac computed tomography: multi-ethnic study of atherosclerosis (MESA). Atherosclerosis 198:104-114

12. Jairam PM, Gondrie M, Grobbee D, Mali W, van der Graaf Y et al (2014) Unrequested imaging findings from routine chest $\mathrm{CT}$ identify subjects at high risk of future cardiovascular events. Radiology 272:700-708

13. Khot UN, Khot MB, Bajzer CT, Sapp SK, Ohman EM et al (2003) Prevalence of conventional risk factors in patients with coronary heart disease. JAMA 290:898-904

14. Nasir K, Rubin J, Blaha MJ, Shaw LJ, Blankstein R et al (2012) Interplay of coronary artery calcification and traditional risk factors for the prediction of all-cause mortality in asymptomatic individuals. Circ Cardiovasc Imaging 5:467-473

15. Silverman MG, Blaha MJ, Krumholz HM, Budoff MJ, Blankstein R et al (2013) Impact of coronary artery calcium on coronary heart disease events in individuals at the extremes of traditional risk factor burden: the Multi-Ethnic Study of Atherosclerosis. Eur Heart J 35:2232-2241

16. Sin DD, MacNee W (2013) Chronic obstructive pulmonary disease and cardiovascular diseases: a "vulnerable" relationship. Am J Respir Crit Care Med 187:2-4

17. Man SF, Van Eeden S, Sin DD (2012) Vascular risk in chronic obstructive pulmonary disease: role of inflammation and other mediators. Can J Cardiol 28:653-661

18. Van Eeden S, Leipsic J, Paul Man SF, Sin DD (2012) The relationship between lung inflammation and cardiovascular disease. Am J Respir Crit Care Med 186:11-16

19. de Jong PA, Gondrie MJ, Buckens CF, Jacobs PC, Mali WP et al (2011) Prediction of cardiovascular events by using non-vascular findings on routine chest CT. PLoS One 6:e26036

20. Prentice RL (1986) On the design of synthetic case-control studies. Biometrics 42:301-310

21. Onland-Moret NC, van der Daphne LA, van der Schouw YT, Buschers W, Elias SG et al (2007) Analysis of case-cohort data: a comparison of different methods. J Clin Epidemiol 60:350-355

22. Jacobs PC, Prokop M, Oen AL, van der Graaf Y, Grobbee DE et al (2010) Semiquantitative assessment of cardiovascular disease markers in multislice computed tomography of the chest: interobserver and intraobserver agreements. J Comput Assist Tomogr 34:279-284

23. Paas GRA, Veenhuizen KCW (2002) Research on the validity of the LMR [in Dutch]. Utrecht: Prismant

24. de Bruin A, Kardaun J, Gast F, de Bruin E, van Sijl M, Verweij G (2004) Record linkage of hospital discharge register with population register: experiences at Statistics Netherlands. Stat J U N Econ Comm Eur 21:23-32

25. Mackenbach JP, van Duyne WM (1984) Registration and coding of various causes of death in the Netherlands and other EEC countries. Ned Tijdschr Geneeskd 128:13-18

26. Merry AH, Boer JM, Schouten LJ, Feskens EJ, Verschuren WM et al (2009) Validity of coronary heart diseases and heart failure based on hospital discharge and mortality data in the Netherlands using the cardiovascular registry Maastricht cohort study. Eur J Epidemiol 24:237-247

27. Light RW (2002) Clinical practice. Pleural effusion. N Engl J Med 346:1971-1977 
28. Nakata M, Saeki H, Takata I, Segawa Y, Mogami H et al (2002) Focal ground-glass opacity detected by low-dose helical CT. Chest 121 : 1464-1467

29. Hansell DM, Bankier AA, MacMahon H, McLoud TC, Muller NL et al (2008) Fleischner Society: glossary of terms for thoracic imaging. Radiology 246:697-722

30. Libby P (2002) Inflammation in atherosclerosis. Nature 420: 868-874

31. Kavousi M, Elias-Smale S, Rutten JH, Leening MJ, Vliegenthart R et al (2012) Evaluation of newer risk markers for coronary heart disease risk classification: a cohort study. Ann Intern Med 156:438-444
32. Pencina MJ, D'Agostino RB Sr, D'Agostino RB Jr, Vasan RS (2008) Evaluating the added predictive ability of a new marker: from area under the ROC curve to reclassification and beyond. Stat Med 27: 157-172, discussion 207-112

33. Kim SS, Seo JB, Lee HY, Nevrekar DV, Forssen AV et al (2013) Chronic obstructive pulmonary disease: lobe-based visual assessment of volumetric CT by using standard images - comparison with quantitative CT and pulmonary function test in the COPDGene study. Radiology 266:626-635

34. Moons KG, Royston P, Vergouwe Y, Grobbee DE, Altman DG (2009) Prognosis and prognostic research: what, why, and how? BMJ 338:b375 